\title{
DIABETES
}

\section{Screening for T2DM does not reduce mortality}

Previous modelling studies have indicated that implementation of a screening programme for type 2 diabetes mellitus (T2DM) could reduce mortality associated with this disease. However, results of an analysis of the first phase of the ADDITION-Cambridge trial suggest that screening does not lead to a reduction in either mortality associated with T2DM or all-cause mortality.

In this unbalanced, cluster-randomized trial, 33 general practices in the east of England were randomized to either inviting or not inviting a predefined list of patients who were at high risk of developing T2DM for a single round of stepwise screening for the disease. Patients from five practices were not invited to participate in the screening programme, and this group of 4,137 individuals comprised the control group. Meanwhile, patients from 13 practices were invited for screening and those identified as having T2DM were followed up with routine care. Patients from a further 15 practices were invited for screening and patients identified as having T2DM were treated intensively. The 16,047 individuals in the latter two groups made up the screening group.

Screening consisted of initial random capillary blood glucose level and $\mathrm{HbA}_{1 \mathrm{c}}$ level measurements, with a confirmatory oral glucose tolerance test performed in patients whose blood glucose level was $\geq 6.1 \mathrm{mmol} / 1$, or whose blood glucose level was $5.5-6 \mathrm{mmol} / 1$ with an $\mathrm{HbA}_{1 c}$ level $\geq 6.1 \%$. Patients were diagnosed as having T2DM in accordance with the 1999 WHO criteria.

No significant difference was found between the two groups in terms of allcause mortality, the primary outcome, over the median follow-up period of 9.6 years (HR 1.06, 95\% CI 0.90-1.25). Additionally, no significant difference was seen between the two groups over the same period for the secondary outcomes of death from cardiovascular disease (HR 1.02, 95\% CI 0.75-1.38), cancers (HR 1.08, 95\% CI 0.901.30 ) and other causes (HR 1.10, 95\% CI 0.87-1.39), or for death related to diabetes mellitus (HR 1.26, 95\% CI 0.75-2.10).
Importantly, thes results were not affected by the subsequent treatment received by individuals who were identified as having T2DM in the groups in which screening took place.

The authors are careful to point out that the population of the area covered by the ADDITION-Cambridge trial does not reflect the whole population of England, being of above-average affluence. Additionally, they acknowledge that the results might have been subject to confounding factors that are not quantifiable, such as the fact that opportunistic screening could have taken place, leading to early diagnosis of patients with T2DM outwith the screening programme under study.

\section{Fiona Mitchell}

Original article Simmons, R. K. et al. Screening for type 2 diabetes and population mortality over 10 years (ADDITION-Cambridge): a cluster-randomised controlled trial. Lancet doi:10.1016/S0140-6736(12)61422-6 